\title{
Predictors of intrauterine growth restriction
}

\author{
Rabindran', Gedam DS ${ }^{2}$ \\ ${ }^{1}$ Dr. Rabindran, Consultant Neonatologist, Billroth Hospital, Chennai, ${ }^{2}$ Dr D Sharad Gedam, Professor of Pediatrics, L N \\ Medical College, Bhopal, MP, India.
}

Address for Correspondence: Dr Rabindran, E mail: rabindranindia@yahoo.co.in

\begin{abstract}
IUGR is pathologic inhibition of intrauterine fetal growth. Risk factors for IUGR comprise maternal factors, fetal factors, adnexal factors \& Placental or umbilical cord factors. For predicting IUGR a combined approach using clinical data, serum markers, biophysical parameters has higher predictive value. Maternal BMI, Symphysis-fundal height have been used to predict IUGR. Biochemical markers are recently being evaluated for predicting IUGR including angiogenesis-related biomarkers, endothelial function/oxidative stress-related biomarkers, placental proteins/ hormone-related biomarkers metabolomics \& genetic biomarkers. Sonography is a noninvasive technique for predicting IUGR early. Sonographically measured fetal femur length- abdominal circumference ratio, Foetal Ponderal Index, Umbilical artery systolic/ diastolic (S/D) ratio, resistance index, pulsatility index, MCA-PSV,TCD/AC ratio, Placental Quotient, increased pulsatility index in uterine artery are used in predicting IUGR. Biometry \& amniotic fluid volume also show promising value in predicting IUGR early.
\end{abstract}

Keywords: IUGR, Biomarkers, Biometry.

Intrauterine growth restriction (IUGR) is defined as pathologic inhibition of intrauterine fetal growth \& failure of fetus to achieve its growth potential. It occurs in about $10 \%$ of pregnancies. Risk factors for IUGR comprise maternal factors like socioeconomic status, weight, smoking, use of recreational drugs, advanced maternal age, nulliparity, history of gestational hypertension, family history of IUGR/ previous IUGR pregnancy, previous pregnancy with preeclampsia, inherited/acquired thrombophilia, anemia, high altitude living, autoimmune disorders, antepartum diabetes mellitus, chronic diseases; fetal factors like multiple gestation, congenital infections, aneuplodies, genetic syndromes; adnexal factors like uterine malformations, subchorionic haematoma, extensive villous infarction, marginal/ velamentous cord insertion, placental mosaicism; Placental or umbilical cord factors like twin-to-twin transfusion syndrome, placental abnormalities, choronic abruption, placenta previa, abnormal cord insertion, cord anomalies. There are various factors associated with IUGR which help in predicting growth restriction early \& hence help in prompt management to prevent stillbirths \& morbidities.

For predicting IUGR a combined approach using clinical data, serum markers, biophysical parameters has higher predictive value. Maternal Body mass index (BMI) screening has been used as an effective method for predicting fetal growth [1]. Symphysis-fundal height

Obsgyne Review: Journal of Obstetrics and Gynecology customized for maternal weight, height \& ethnicity has nearly $50 \%$ detection rate for IUGR screening. A lag in fundal height of $4 \mathrm{~cm}$ or more suggests IUGR. Biochemical markers studied in association with IUGR include pregnancy associated plasma protein A (PAPPA), alpha fetoprotein, $\beta-\mathrm{hCG}$, unconjugated estriol, inhibin-A, A-disintegrin and metalloprotease 12 (ADAM12), placental protein 13 (PP-13) [2]. However sensitivity of PAPP-A \& $\beta$-hCG as isolated screening tools is low. Integrated assessment of PP-13, PAPP-A \& uterine artery doppler at 11-13 weeks of gestation has a $20 \%$ detection rate of IUGR [3]. Newer biomarkers recently being evaluated for predicting IUGR include angiogenesisrelated biomarkers like placental growth factor (plgf), soluble fms-like tyrosine kinase-1(sflt-1), soluble endoglin, vascular endothelial growth factor; endothelial function/oxidative stress-related biomarkers like homocysteine, leptin, asymmetric dimethyl-arginine, soluble vascular cell adhesion molecule-1, interferon-C, C-reactive protein, folate; placental proteins/ hormonerelated biomarkers like Insulin-like growth factor binding protein-1 \& -3, Activin A, placental growth hormone; metabolomics \& genetic biomarkers.

Sonography is a noninvasive technique for predicting IUGR early. Sonographically measured fetal femur length- abdominal circumference ratio is a date independent predictor of IUGR helpful in evaluating high-

Available online at : www.medresearch.in 1 | P a g e 
Editorial

risk patients presenting late with no dates. Foetal Ponderal Index (PI) is a predictor of IUGR with sensitivity around $75 \%$ \& specificity around $80 \%$ [4]. Umbilical artery systolic/diastolic (S/D) ratio, resistance index (RI) \& pulsatility index (PI) are used commonly to detect IUGR. An average $\mathrm{S} / \mathrm{D}$ ratio $>3$ at 30 or more weeks of gestation has a sensitivity of $78 \%$ \& specificity of $85 \%$ in predicting IUGR. However Umbilical artery doppler early between 10-14 weeks of gestation has a low positive predictive values for IUGR but better prediction for IUGR secondary to preeclampsia or IUGR alone with delivery < 32 weeks. Middle cerebral artery -peak systolic velocity (MCA-PSV) is a better predictor of IUGR. Transverse cerebellar diameter/ Abdominal circumference (TCD/AC) ratio exceeding $2 \mathrm{SD}$ above mean is predictive of growth restriction with both sensitivity\& specificity around $70 \%$. Placental Quotient (PQ) [placental volume / crown rump length] assessed with uterine artery doppler has low sensitivity in diagnosis of IUGR. During $2^{\text {nd }}$ trimester IUGR in low-risk patients is best predicted by an increased pulsatility index in uterine artery with notching (positive likelihood ratio 9.1)[5]. Recently virtual organ computer-aided analysis software (VOCAL) using vascularization index (VI), flow index (FI) \& vascularization flow index (VFI) detect changes in vascularisation earlier than umbilical artery doppler abnormalities.

Biometry \& amniotic fluid volume have been used to predict IUGR. Low abdominal circumference (AC) percentile has highest sensitivity (98\%) for diagnosing IUGR [6]. Amniotic fluid maximum vertical pocket (MVP) value $<2 \mathrm{~cm}$ was associated with IUGR rate of $20 \%$ whereas MVP $<1 \mathrm{~cm}$ with an IUGR rate around $40 \%$ [7]. Using oligohydramnios definition of largest vertical fluid pocket $<1 \mathrm{~cm}$, Manning reported sensitivity $\&$ specificity for prediction of IUGR around $84 \%$ \& 97\% respectively [8]. Total intrauterine volume (TIUV) has also been used for predicting IUGR. 3-Dimensional ultrasonography has been used to measure fetal femur or fetal humerus volume as a predictor of IUGR. Measurement of fetal soft tissue like subcutaneous tissue thickness at level of fetal midcalf, midthigh or abdominal wall \& cheek-to-cheek diameter is probably predictive of IUGR with sensitivity \& specificity around $76 \& 67 \%$ respectively [9]. Maternal plasma fibronection at a cut point of $475 \mathrm{mg} / \mathrm{L} \&$ serum ferritin are recently being used as predictors of IUGR [10]. With advances of medical field \& rise of newer biochemical markers \& imaging techniques it is possible to predict IUGR early \& thereby prevent stillbirths \& neonatal morbidities.

\section{References}

1. Nahar S, Mascie-Taylor CG, Begum HA: Maternal anthropometry as a predictor of birth weight. Public Health Nutr. 2007 Sep;10(9):965-70.DOI. 10.1017/ S1368980007217975.

2. Gagnon A, Wilson RD, Audibert F, Allen VM, Blight C, Brock JA, Désilets VA, Johnson JA, Langlois S, Summers A, Wyatt P. Obstetrical complications associated with abnormal maternal serum markers analytes J Obstet Gynaecol Can. 2008 Oct;30(10):918-49.

3. Poon LC, Syngelaki A, Akolekar R, Lai J, Nicolaides $\mathrm{KH}$. Combined screening for preeclampsia and small for gestational age at 11-13 weeks. Fetal Diagn Ther. 2013;33(1):16-27. doi: 10.1159/000341712.

4.Vingt LAM. Lodeiro JG, Feins SJ. Value of fetal ponderal index in predicting growth retardation. Obstet Gynecol 1986; 67:584-8.

5. Jeltsje S. Cnossen, Rachel K. Morris, Gerben ter Riet,et al., Use of uterine artery Doppler ultrasonography to predict pre-eclampsia and intrauterine growth restriction: a systematic review and bivariable meta-analysis. CMAJ. 2008 Mar11;178(6): 701-711. doi: 10.1503/cmaj.070430.

6. Yoshida S, Unno N, Kagawa H, Shinozuka N, Kozuma S, Taketani Y.Prenatal detection of a high-risk group for intrauterine growth restriction based on sonographic fetal biometry. Int J Gynaecol Obstet. 2000 Mar;68(3):225-32.

7. Banks EH, Miller DA. Perinatal risks associated with borderline amniotic fluid index. Am J Obstet Gynecol. 1999 Jun;180(6 Pt 1):1461-3.

8. Manning FA, Hill LM, Platt LD. Qualitative amniotic fluid volume determination by ultrasound: antepartum detection of intrauterine growth retardation. Am J Obstet Gynecol. 1981 Feb 1;139(3):254-8.

9. Hill LM, Guzick D, Thomas ML, et al. Thigh circumference in the detection of intrauterine growth retardation. Am J Perinatol 1989; 6:349.

10.Wang Zehua, Xiong Guirong, Zhu Ying.Thepredictive value of plasma fibronectin concentration on fetal growth retardation at earlier stage of the third trimester. Journal of Tongji Medical University; September 2001, Volume 21, Issue 3, pp 253-255.

\section{How to cite this article?}

Rabindran, Gedam DS. Predictors of intrauterine growth restriction. Obs Rev:J obstet Gynecol 2016;2(2):1-2.doi: 10.17511/joog.2016.i02.01. 